INTERNATIONAL JOURNAL OF FILM AND MEDIA ARTS (2020) Vol. 6, №. 1 pp. 55-72

(c) 2021 BY-NC

ijfma.ulusofona.pt

doi: 10.24140/ijfma.v6.n1.03

\title{
TECHNO-ANIMISM \\ OR THE MAGICAL \\ EXISTENCE OF \\ TECHNICAL OBJECTS
}

MANUEL BOGALHEIRO* 
INTERNATIONAL JOURNAL OF FILM AND MEDIA ARTS (2021) Vol. 6, №. 1

Manuel Bogalheiro teaches in the Faculty of Communication, Architecture, Arts and Information Technologies at Lusófona University of Porto, where he coordinates the PhD in Media Arts (ULP/ULHT with Victor Flores). He has a PhD in Communication Sciences - Contemporary Culture and New Technologies (FCSH-UNL), with a thesis entitled "Materiality and Technicity: On the Technical Objectuality". He was a FCT research fellow. He researches and publishes in the fields of philosophy of technics, media theory and culture.

\section{Corresponding Author}

manuel.bogalheiro@ulp.pt

FCAATI/ Lusófona University

Rua Augusto Rosa, 24

4000-098 Porto

Portugal

Paper submitted: 29th November 2020

Accepted for Publication. 26th April 2021

Published online: $9^{\text {th }}$ June 2021 


\begin{abstract}
If, on the one hand, the classic notion of artefact presupposes inert objects and a reliability between the user and the uses that can be extracted from the objects, on the other hand, it is recognized a suspicion that technical objects and their operative schemes, the machines and the "artificial" solutions contain in themselves an inauthentic and adulterating nature, a power of cunning and trickery capable of emancipate and process in autonomous forms, regardless of the uses and apprehensions that humans, their operators, may make. The western imaginary of technics would thus have been constituted under presuppositions that, together with the formal laws of mechanics or the cold mathematics that governs any grammar of codes, projected a animistic and a magical dimension in all the artificiality that allowed to manipulate the original productivity of nature.
\end{abstract}

With the emergence of digital technologies and the general informatization of artefacts, this dimension took shape in what Philip K. Dick would consider to be a techno-animism tendency and that ubiquitous computing and internet of things would materialize in the era of so-called cognitive capitalism.

In this paper, we seek to approach techno-animism as a speculative aesthetics that, by projecting a life into technical objects, inscribes in them an alterity and a strangeness that could enhance other perspectives beyond the usual uses and relationships that we take for granted and that hide objects, as well as the political or commercial strategies that mobilize them. Enchanting technical objects is, on the one hand, creating a possibility to suspend them from their instrumental flows and, on the other, installing a weird or magical realism that disturbs the articulations of a mere and exclusively human reality.

Keywords: animism, technical object, agency, speculative aesthetics, magical realism

\title{
Tecno-animismo ou a existência mágica de objetos técnicos
}

\section{Resumo}

Se, por um lado, a noção clássica de artefacto pressupõe objetos inertes e uma confiabilidade entre o usuário e os usos que podem ser extraídos dos objetos, por outro lado, reconhece-se a suspeita de que os objectos técnicos e os seus esquemas operatórios, as máquinas e as suas soluções "artificiais", encerram em si um carácter inautêntico e adulterador, como se de uma potência própria de astúcia e de engano se tratasse, capaz de, no limite, se emancipar e se processar de forma autónoma, independentemente dos usos e das apreensões que os humanos, seus operadores, possam fazer. O imaginário ocidental da técnica ter-se-á assim constituído sob pressupostos que, a par das leis formais da mecânica ou da matematicidade fria que governa qualquer gramática de códigos, projectaram uma dimensão mágica e especulativa naquilo que de artificial permite manipular a produtividade original da natureza.

Com o surgimento das tecnologias digitais e a informatização geral dos artefactos, essa dimensão adquiriu forma naquilo que Philip K. Dick considerou a tendência do tecno-animismo e que, mais tarde, seria materialmente concretizada pela computação ubíqua e pela internet das coisas na era do chamado capitalismo cognitivo.

Neste artigo, procuramos abordar o tecno-animismo como uma estética especulativa que, ao projectar uma vida própria nos objectos técnicos, Ihes inscreve uma alteridade e uma estranheza que potencia perspectivas para além dos usos e relações habituais que se dão por garantidas e que escondem os objectos, assim como para além das estratégias políticas ou comerciais que os mobilizam. Encantar objetos técnicos é, por um lado, criar uma possibilidade para os suspender dos seus fluxos instrumentais e, por outro, instalar um realismo mágico ou problemático que perturba as articulações de uma realidade mera e exclusivamente humana.

Palavras-chave: animismo, objecto técnico, agenciamento, estética especulativa, realismo mágico.. 
Don't content yourself with examining the innermost voices and the most hidden motives of our own soul (a luxury lacking consequences) but do examine the secret voices, motives, and maxims of your instruments.

\section{Günther Anders ${ }^{1}$}

\section{Dos artefactos às máquinas: a suspeita original}

A história ocidental da técnica terá em grande medida assentado numa concepção dos artefactos que os projectou como objectos estáveis e controlados, decididos e localizáveis, contínuos na relação entre o seu design físico e a sua utilidade, analógicos na relação entre a sua manipulação material e os seus efeitos. Nesta concepção, foi atribuído aos artefactos um papel de estabilização, de segurança e de confiabilidade, perante o irremediável composto de mudança e de indeterminação que sempre atravessou a experiência e que tudo ameaça como fugaz e efémero: os objectos como pontos de excepção no real, pontos consistentes aos quais se pode sempre voltar com a mesma certeza, reconhecendo a identidade do que persiste e abrandando os impulsos do tempo. A história dos artefactos é, neste sentido, a história de diversas formas através das quais a experiência é estabilizável.

As coisas do mundo têm a função de estabilizar a vida humana; a sua objectividade reside no facto de que - em contradição com o dito de Heraclito de que o mesmo homem nunca pode entrar no mesmo rio os homens, apesar da sua contínua mutação, podem reaver a sua invariabilidade, isto é, a sua identidade, nos objectos que não variam, como a mesma cadeira e a mesma mesa (Arendt, 1998 [1959], p. 137). ${ }^{2}$

A tal concepção correspondeu uma estrutura de mapeamento do real e dos seus objectos de acordo com os referentes e com as regularidades da natureza dada - forma, matéria, eficiência, fins, espaço, tempo, consistência física. Em certa medida, é nessas categorias - que reflectem o modelo clássico da instrumentalidade - que se baseia o modelo ocidental da produção: distinguir o natural do artificial, mas também o poder do criador ou do operador da passividade dos meios que são subordinados a fins previstos e da matéria inerte que é trabalhada e enformada.

As origens deste modelo clássico da produção podem ser reconhecidas na célebre definição aristotélica de artefacto técnico como qualquer coisa que "não tem um impulso inato para a mudança" (Aristóteles, Física, 192b - 193b), ou seja, qualquer coisa que é fundamentalmente inerte e neutra, sem devir imanente ou sem capacidade para se mobilizar a si própria. A natureza essencial dos artefactos é ser da ordem das coisas inanimadas, incapazes, portanto, de se produzirem e reproduzirem a si próprias. Como produtos da techné e enquanto manifestação da criação humana, os artefactos distinguem-se do impulso auto-poiético da natureza e de todas as coisas que existindo por natureza - isto é, tendo princípio e causa na própria physis - apresentam em si um "princípio de mudança e de não mudança - seja no que toca ao lugar, ao crescimento, à decadência ou à alteração qualitativa" (Aristóteles, Física, 192b - 193b). Uma cama, para recorrer ao exemplo dado por Aristóteles na mesma secção da Física, pode apodrecer, expondo em si o princípio da mudança, mas apenas por ser feita de madeira. É pela sua natureza material que a transformação pode ocorrer, não pela sua forma ou

1 Anders, G. (1961). [1957]. "Commandments in the Atomic Age." Burning Conscience. Monthly Review Press

2 Também Michel Serres, num livro dedicado à dialéctica entre as formas históricas da ordem e as da desordem, desenvolve uma posição próxima da de Hannah Arendt: "The only assignable difference between animal societies and our own resides in the emergence of the object. Our relationships, social bonds, would be airy as clouds were there only contracts between subjects. In fact, the object, specific to the Hominidae, stabilizes our relationships, it slows down the time of our revolutions. The object, for us, makes our history slow" (Serres, 1985, p. 87). 
pela sua essência ou, dito de outro modo, a mudança dá-se por causa daquilo que é feito um artefacto e não por aquilo que ele é.

A distinção aristotélica funda a ontologia ocidental sobre a qual se sustenta a divisão originária entre o natural e o artificial. ${ }^{3}$ Por não se constituir por uma natureza própria, nem pelo acaso ou pelo espontâneo, mas por uma acção humana e por um procedimento técnico, a existência dos artefactos fica refém de uma efectividade que Ihes é exterior, para serem originalmente produzidos, para serem reproduzidos e para cumprirem a função própria que lhes compete e para a qual foram produzidos. Sem princípio intrínseco, "um objecto é um artefacto se e somente tiver um autor" (Hilpinen, 2004) e, acrescente-se, a sua existência apenas se justifica enquanto cumprir devida e fielmente a funcionalidade que Ihe foi inscrita pelo seu criador sem levantar entraves. ${ }^{4}$ Como numa espécie de dívida irredutível, apenas perante um sujeito os artefactos podem ser activados: do artífice que os produz ao utilizador que os manuseia, rege o seu funcionamento, cristaliza os seus usos e controla a sua potência, ou, enfim, aos sistemas culturais que lhe definem o estatuto e acabam por determinar a sua obsolescência. Neste entendimento, os artefactos são despojados de qualquer impulso congénito e reduzidos a meros meios para fins, dependendo de um sujeito que os operacionalize e rentabilize. Como Bernard Stiegler assinala, não há, nesta ontologia, o reconhecimento da possibilidade de uma autodeterminação ou causalidade própria dos seres técnicos (Stiegler, 1998, p. 1), pressuposto que não deixa de encerrar em si uma dimensão política: tal como o escravo não existe sem o senhor, também os artefactos não existem sem o seu criador ou operador. ${ }^{5} \mathrm{E}$ é nesta subalternidade ontológica atribuída aos artefactos e aos objectos técnicos que se pode projectar historicamente no domínio do artificial e da técnica um domínio de segurança e de certezas, no qual a confiabilidade geral nos artefactos não dispõe a que, aquele que os utiliza, possa ser surpreendido ou traído por uma vitalidade autónoma.

Tal cenário não deixa, no entanto, de ser especulado por Aristóteles num exercício de pensamento que surge noutro contexto e, ainda que fora dos problemas da física, não deixa de reflectir o problema ontológico em causa:

Se cada instrumento pudesse desempenhar a sua função a nosso mando, ou como que antecipando-se ao que se lhe vai pedir - tal como se afirma das estátuas de Dédalo ou dos tripés de Hefesto acerca dos quais o poeta diz movendo-se por si mesmas entram na assembleia dos deuses - e se, do mesmo modo, os teares tecessem sozinhos, e se as palhetas tocassem sozinhas a cítara, então os mestres não teriam necessidade de ajudantes nem os senhores de escravos (Aristóteles, Política, 1255b, 35).

Mesmo que seja da ordem de um exercício de pensamento, a passagem de Aristóteles aponta para outras possíveis dimensões dos artefactos para além daquelas que o seu dispositivo original da produção Ihes confere. Desde logo, a possibilidade de uma automatização que tendencialmente

3 As teses da tecnicidade originária procuram dissolver esta divisão. Cf. Bradley, (2011).

4 Vilém Flusser, teórico materialista do design, foi sempre contestando esta posição. Veja-se, por exemplo: "Um simples 'objecto' é algo que estorva, um obstáculo que foi 'lançado' no nosso caminho (em grego problema). O mundo é objectivo e problemático, na medida em que constitui um obstáculo. Um 'objecto de uso' é um objecto necessário à remoção de outros objetos do seu caminho. Esta definição encerra uma contradição; um obstáculo que serve para remover obstáculos?" (Flusser, 2010, p. 57).

5 Gilbert Simondon verá neste princípio a origem de uma atitude na cultura ocidental de discriminação ou mesmo, como refere, de "escravatura" dos objecto: "la culture se conduit envers l'objet technique comme l'homme envers l'étranger quand il se laisse emporter par la xénophobie primitive. Le misonéisme orienté contre les machines n'est pas tant haine du nouveau que refus de la réalité étrangère" (Simondon, 2012, p. 10). Voltaremos mais à frente ao pensamento do filósofo francês. 
se transformaria em autonomização e, para além disso, a hipótese de que na natureza dos artefactos há um agenciamento maquínico que a qualquer momento se pode revelar, de modo imprevisível, com uma astúcia própria e com energias que não foram contempladas no seu design "exterior". ${ }^{6}$ Do que aqui se trata é da possibilidade de desvio dos artefactos em relação ao plano antropomórfico a partir do qual foram idealizados. Na medida em que a sua acção concreta sempre se pode revelar mais complexa do que a sua gramática inicial de operações previu, os artefactos serão actantes, os quais, como Bruno Latour assinala, ao "serem mobilizados para garantir uma aliança não deixam de agir em seu próprio nome; cada um continua a fomentar os seus próprios planos, formando os seus próprios grupos e servindo a outros mestres, vontades e funções" (1988, p. 197). Se a possibilidade mais radical, e porventura mais improvável, aponta para que os artefactos dispensem os seus operadores, na hipótese de um agenciamento maquínico dos artefactos está em causa algo mais subtil, mas não menos decisivo, algo que não será tanto da ordem da ameaça, mas sobretudo de uma suspeita: sempre que activados ou postos em funcionamento os artefactos e os seus esquemas operatórios - ou as máquinas e as suas soluções artificiais - encerram em si uma operacionalidade própria que escapa ao controlo ou à apreensão daquele que os manuseia ou opera.

Se a posição de Aristóteles deposita uma relação de confiança nos artefactos produzidos, coexiste nos gregos antigos uma outra posição que - não sendo tanto relativa aos objectos, mas sobretudo relativa ao que podemos chamar uma processualidade técnica - teme os efeitos da techné e vê na sua operacionalidade uma potência adulteradora. Referimo-nos àquilo que está em questão na crítica platónica à técnica da escrita enquanto Pharmakon - remédio ou veneno - e Hypomnesis que - ao contrário da memória interior, a
Anamnese, a voz viva e pura, verdadeiramente livre por não ser objecto de nenhuma mediação técnica e, assim, ser expressão da redescoberta perpétua da verdade pela alma imortal - corresponde a uma empobrecida e mortificada rememoração artificial, refém dos suportes materiais - das extensões - que enfraquecem e definham as faculdades humanas originais (Platão, Fedro, 274d - 275b). Confiar na escrita terá como efeito dramático o esquecimento. A técnica da escrita é, em Platão, um simulacrum na sua acepção pejorativa, isto é, algo que se opõe ao espontâneo, ao intuitivo, ao orgânico ou ao natural. Enquanto techné de empréstimo, e a suspeita reside aqui, a técnica da escrita apenas pode restituir de forma inexacta e adulterada aquilo que apenas pode ter a sua expressão autêntica no diálogo vivo. Deste modo, a crítica que Platão apresenta no Fedro tem como última consequência um entendimento da técnica da escrita como qualquer coisa inumana, um produto manufacturado, que pretende estabelecer fora da mente aquilo que na verdade apenas pode estar na mente. Numa imagem, a mão que escreve cede parte da autonomia do sujeito a um medium exterior que, em certa medida, Ihe é estranho, que não é confiável e que tem as suas próprias regras e materialidades. Trata-se de um artifício cujo domínio, dado que não é natural, tem de ser aprendido para, num movimento irreversível, passar a dominar e a constituir a experiência da expressão, das gestualidades aos modos de formulação do pensamento.

Poder-se-á assumir que na análise platónica se reconhece esse aspecto paradoxal de, pela atenção dada ao artifício que é objecto de crítica, se legitimar a sua potência, o seu poder enquanto a priori técnico da experiência humana, relativizando, sob uma suspeita e uma ameaça, o primado do operador sobre o meio. Mas será precisamente por se reconhecer tal poder que a técnica que o produz deverá ser encarada com desconfiança. A processualidade da escrita faz de si tanto

6 "The production of any artifact is never simply a matter of envisioning some model in thought and then fashioning matter according to that form. While the craftsman's intentions and map play a role in the production of the artifact, the things themselves, the matter used, the circumstances under which they're produced, all contribute to the final product in ways not anticipated by the craftsman" (Bryant, 2014, p. 22). 
um princípio mecânico como uma máquina propriamente dita: "cada máquina declara o que faz: 'este é o modo através do qual esta acção pode ser realizada'. Uma técnica, ou procedimento técnico, é uma escrita na medida em que segue sua própria técnica, seguindo as suas próprias trajectórias" (Connor, 2016, pp. 18-19). ${ }^{7}$

Na perspectiva platónica podemos reconhecer os fundamentos de um certo cepticismo em relação à técnica que, se em última instância resultará em perspectivas negacionistas e tecnofóbicas ou em acções ludistas, num nível mais imediato, e de modo ambivalente, está relacionado com uma certa mistificação - um encantamento - de tudo aquilo que, não sendo reconhecido na natureza nem na acção directa humana, produz, distorce, filtra e faz aparecer pelos seus próprios meios. Não será por acaso que, de acordo com a análise de Jessica Wolfe (2004), a semântica moderna do conceito de tecnologia esteja marcada, no século XVI, por sentidos como os de astúcia, impostura ou fraude, aspecto que mais do que apontar para uma crítica da técnica, assinala uma espécie de ilusionismo natural contido nos aparelhos:

Galileu e Bacon olham para os instrumentos mecânicos como uma forma de transcender os erros e limitações dos sentidos, mas também estão cientes de que as máquinas, como a 'luz incerta dos sentidos' e a imaginação, distorcem as informações que transmitem. (...) A maquinaria imita, em vez de corrigir, os erros e as vaidades dos nossos sentidos (Wolfe, 2004, pp. 4 - 5).

Para além deste aspecto, na mais elementar das implicações do encantamento da técnica, a produtividade espontânea e auto-poiética que estava circunscrita no domínio da natureza parece, então, poder realizar-se por vias não naturais. 0 artificial simula a produtividade da natureza e quanto maior for a sua eficácia mais o modelo clássico da produção é perturbado, na medida em que, no último horizonte, o trabalho, enquanto exercício que implica um "custo" e um "esforço", pode desaparecer.

Todas as actividades produtivas são medidas contra o padrão mágico [magic standard], isto é, a possibilidade de que o mesmo produto possa ser produzido sem esforço, e a eficácia relativa das técnicas é uma função da extensão em que convergem para o padrão mágico de trabalho zero para o mesmo produto. (...) Se há alguma verdade nesta ideia, então podemos ver que a noção de magia, como meio de garantir um produto sem o custo do trabalho que ele realmente acarreta, utilizando os meios técnicos prevalecentes, é efectivamente construída na avaliação padrão que é aplicada à eficácia das técnicas. (...) A magia é a base contra a qual o conceito da obra enquanto custo toma forma. (...) A magia assombra a actividade técnica como uma sombra; ou melhor, a magia é o contorno negativo do trabalho (...) A magia é o meio ideal de produção técnica. (Gell, 1999, pp. 179 - 180)

A possibilidade de uma causalidade e efectividade próprias da técnica obriga a uma redefinição do modelo clássico da produção segundo o qual apenas o humano e a natureza produzem por si mesmos. Entre o deslumbramento e o temor, a libertação e a alienação, o empoderamento e a exclusão, o aspecto mais inquietante do universo da técnica residirá no seu poder demiúrgico para produzir ou fazer aparecer sem

7 "If all writing is a kind of machinery, why might it be plausible to see every machine as a kind of writing? Because every mechanical or technical action can be seen as a procedure as well as a mere proceeding, where a procedure means a replicable operation. So a technical procedure is the styling of a process and, as such, the declarative performance of that process as iterable procedure. Every machine declares of what it does: 'this is the way this action may be performed'. A technic, or technical procedure, is a writing in that it tracks its own technique, following in its own tracks" (Connor, 2016, pp. 18-19). 
esforço, numa espécie de automatismo alquímico, cujo ritmo célere e a processualidade própria ${ }^{8}$ parecem exceder qualquer tentativa de racionalização absoluta do mecanismo. É neste aspecto que a hipótese especulativa da eficácia total das máquinas e das suas operações adquire uma dimensão mágica: como uma potência enigmática e perturbadora, ao mesmo tempo que é da ordem de uma sedução manipuladora, a técnica carrega em si a centelha da magia na medida em que, também, é projectada como um campo de acção de forças superiores em relação às quais os humanos são parcial ou completamente ignorantes.

Com a Modernidade Industrial dos séculos XVIII e XIX, esta percepção mistificadora do fazer técnico conhece um momento fulgurante. As máquinas multiplicam-se em massa e cumprem finalmente a promessa da sua efectiva automatização. ${ }^{9}$ Os efeitos mais imediatos desta revolução ter-se-ão plasmado naquilo que mais do que uma suspeita passa a ser uma ameaça: os homens serão substituídos pelas máquinas. ${ }^{10}$ Como escreve Hannah Arendt "até mesmo a mais sofisticada ferramenta permanece como serva, incapaz de guiar ou substituir a mão; por outro lado, até mesmo a mais primitiva das máquinas guia o labor do nosso corpo até o substituir inteiramente" (Arendt, 1998, p. 157). Em sintonia com esta ideia, Gilbert Simondon nota que, depois do tear automático de cartões perfurados inventado por Joseph-Marie Jacquard ${ }^{11}$ - objecto da fúria dos ludistas -, o homem "torna-se apenas espectador dos resultados de funcionamento das máquinas" (Simondon, 2012, p. 163). No entanto, para além deste aspecto particular, no seio desta ameaça - a qual não deixa de corresponder a uma fase de libertação própria das máquinas depois da interdição antiga a que tinham sido condenadas como seres inertes e servis - joga-se uma implicação mais profunda: a suspeita original em relação ao operar ou ao aparecer técnicos - seja em entes, seja em processos magicamente animados - transforma-se num imaginário personificado no qual as máquinas adquiririam vida própria em corporificações animadas entre traços antropomórficos e traços alienígenas. Hegel dá-se conta deste aspecto quando se refere ao universo das máquinas como "um sistema monstruoso de comunidade e interdependência, uma vida autónoma da matéria morta que, como um animal selvagem, precisa de uma firme e apertada dominação e domesticação". (as cited in Rodrigo, 2014, p. 16).

É neste imaginário animista que, por exemplo, poderemos enquadrar a máquina que Novalis conjectura em 1799 descrita como "um moinho monstruoso, movido pelo fluxo do acaso e flutuando sobre ele, um moinho de si mesmo sem construtor ou sem moeiro, um verdadeiro perpetuum mobile, um moinho a moer-se a si próprio" (1997, p. 144). Ou, já não na esfera do teratológico vitalista, mas no campo de possibilidades de um cérebro artificial, e sob a inspiração da Máquina Analítica (1837) de Charles Babbage, poderíamos também reconhecer nesta perspectiva os autómatos inteligentes personificados

8 Diderot refere-se assim a esta ideia: "a natureza leva séculos a preparar grosseiramente os metais; a techné propõe aperfeiçoá-los num dia. A natureza leva séculos a formar as pedras preciosas, a arte reclama que as reproduz num instante" (Diderot, 1753, p. 14).

9 "The magic of automation [is] literal minded... A goal-seeking mechanism will not necessarily seek our goals unless we design it for that purpose, and in that designing we must foresee all steps of the process for which it is designed... The penalties for errors of foresight, great as they are now, will be enormously increased as automatization comes into its full use" (Wiener, 1966, p. 63).

10 "The machine, therefore, is a mechanism, that, after being set in motion, performs with its tools the same operations as the worker formerly did with similar tools. Whether the motive power is derived from man, or in turn from a machine, makes no difference here. From the moment that the tool proper is taken from man, and fitted into a mechanism, a machine takes the place of a mere implement. The difference strikes one at once, even in those cases where man himself continues to be the prime mover. The number of implements that he himself can use simultaneously is limited by the number of his own natural instruments of production, i.e. his own bodily organs." (Marx, 1990, p. 495)

11 São abundantes os exercícios arqueológicos que identificam no tear de Jacquard a origem da programação e, consequentemente, dos computadores. Confira-se, por exemplo: Essinger, 2004 
no jogadores de xadrez descritos por Edgar Allan Poe em 1836 ou por Ambrose Bierce em 1894. ${ }^{12}$

\section{O tecno-animismo e a informatização geral dos objectos}

A persistência desta mistificação animista, identificável no seio da própria técnica, parece antagónica ao espírito da Modernidade tecno-científica assente nas leis formais da mecânica ou na matematicidade que governa qualquer gramática de códigos e procura dar exactidão ao mundo, aperfeiçoando-o cumulativamente na mesma medida em que o liberta do mítico, do teológico ou do metafísico. Esse espírito sustenta-se através de uma narrativa do progresso na qual, como assinala o antropólogo Alfred Gell, o "homo technologicus é uma criatura racional, sensível, e não mitopoética ou religiosa, à qual apenas regressa quando abandona a busca de soluções 'técnicas' para os seus problemas e se retira para os domínios da fantasia e da especulação vazia" (Gell, 1988, p. 6). À luz deste pressuposto, o animismo e a magia apenas persistem na forma de uma perturbação ou da ameaça de um retrocesso a um estádio primitivo. No entanto, como o próprio Gell logo acrescenta, "essa oposição entre o técnico e o mágico não tem fundamento" (1988, p. 6). Procuraremos, mais à frente, justificar a importância, ou mesmo a necessidade, desta relação. Para já, importa-nos assinalar como é que, depois da Modernidade Industrial - e considerando a fase cibernética da técnica da segunda metade do século XX, na qual a energia das máquinas industriais e a matemática das primeiras máquinas analíticas se fundem em arquitecturas e redes informacionais e computacionais - esta tendência animista não se terá apenas perpetuado, como é até reconhecida com sinais mais evidentes.

Philip K. Dick, numa conferência de 1972 intitulada "O Androide e o Humano", começa por assinalar que a psicologia moderna assenta numa tentativa de anular essa pulsão primitiva relativa a animar os objectos que nos rodeiam, tal como se tivessem um propósito ou uma vida própria. Essa anulação seria, do ponto de vista civilizacional, condição para a maturidade, a sanidade e também a cientificidade do homem moderno. Em suma, trata-se aqui, nesta disposição clínica, de mortificar o mundo para reforçar a vida do humano. K. Dick critica tal tendência través de dois argumentos. Primeiro, ao afirmar que esse processo de reificação do mundo carrega em si o risco de passar dos objectos para os próprios sujeitos, acabando estes últimos também reificados. Segundo, ao realçar que o mundo das tecnologias de informação e das redes computacionais, mesmo que codificadas em modelos cada vez mais abstractos e numéricos, expressa irredutivelmente uma dimensão "primitiva", reabilitando a percepção de uma realidade animada por objectos técnicos vivos e pulsantes. O seu diagnóstico é exemplar perante um mundo digital que entretanto se complexificava e se absolutizava na segunda metade do século XX:

O nosso ambiente - e refiro-me ao nosso mundo artificial de máquinas, construções, computadores,

12 Tratam-se de dois célebres contos sobre a hipótese de as máquinas pensarem. Se o conto de Poe (2015) direcciona a questão para o problema da "abertura" dos máquinas, isto é, se os autómatos apenas cumprem um programa matemático fechado e automático ou se podem, de alguma forma, aprender e desenvolverem-se a si mesmos a partir dos princípios mínimos desse programa, o conto de Bierce (2011) direcciona o problema para um horizonte mais largo relativo à hipótese de um animismo geral, um agenciamento vitalista que seria comum ao humano, ao vegetal, ao geológico e também ao maquínico. "All matter is sentient, every atom is a living, feeling, conscious being. There is no such thing as dead, inert matter: it is all alive; all instinct with force, actual and potential; all sensitive to the same forces in its environment and susceptible to the contagion of higher and subtler ones residing in such superior organisms as it may be brought into relation with, as those of man when he is fashioning it into an instrument of his will. It absorbs something of his intelligence and purpose-more of them in proportion to the complexity of the resulting machine and that of its work. (...) In the definition of 'life' the activity of a machine is included there is nothing in the definition that is not applicable to it. According to this sharpest of observers and deepest of thinkers, if a man during his period of activity is alive, so is a machine when in operation." (Bierce, 2011, pp. $254-255$ ). 
sistema electrónicos, componentes homeostáticos interligados entre si - começa a adquirir aquilo que os psicólogos receiam que o primitivo veja no seu ambiente: vida. Num sentido muito palpável, o nosso ambiente está a tornar-se vivo, ou quase-vivo, de formas específica e fundamentalmente análogas a nós (Dick, 2006, p. 30).

Neste diagnóstico, ${ }^{13}$ no qual se plasma uma restituição possível da potência da vida às produções, supostamente inertes, da técnica, está em causa uma aproximação entre o técnico e o orgânico ou até o humano, assim como a possibilidade de - em vez de reduzidos à positividade fáctica das suas partes funcionais ou a leituras estritamente mecanicistas - perspectivar a realidade técnica através de um vitalismo no qual reside a condição da sua legitimação. Como escreve K. Dick, "é agora possível estudar o nosso ambiente artificial, de que modo se comporta, por que o faz e o que procura fazer, por analogia com aquilo que sabemos sobre nós próprios" (2006, p. 31). Em todo o caso, este será apenas um passo inicial para uma outra perspectiva sobre a técnica que, no entanto, não poderá esgotar totalmente os seus mecanismos e as suas processualidades. É que se o pensamento analógico, e não dualista entre técnico e humano, será positivo para uma melhor integração da tecnologia na cultura - não enquanto obstáculo ou simples domínio de exploração -, os produtos da técnica guardarão sempre uma margem própria na qual se expressa a sua singularidade. Será neste sentido que o escritor afirma que "os artefactos não imitam os humanos; eles já são, em muitos sentidos profundos, humanos de facto" (2006, p. 32). Surge, então, um novo antropomorfismo, já não a partir das formas humanas projectadas - o seu aspecto corporal, os seus traços físicos, as suas gestualidades - noutros entes, mas a partir da inteligência, da personalidade e, no limite, da consciência humanas. ${ }^{14}$ É certo que nesta inversão em que, de alguma forma, as máquinas são humanizadas, não deixa de existir qualquer coisa de perturbador e a "sensação desconfortável de que estas coisas estão a tentar fazer-se passar por seres humanos sem o serem" (Dick, 2006, p. 77). Pese embora esta ressalva, o mais importante para K. Dick nesta transição será - nos termos de uma cosmologia pré-moderna ou de um tecno-paganismo que reencanta os objectos técnicos contemporâneos à luz dos primeiros e arcaicos ossos talismânicos - uma crítica à reificação civilizacional da experiência contemporânea. Numa procura para anular, ou complexificar, o dualismo clássico entre homem e máquina, afırma que "talvez estejamos a assistir a uma fusão gradual da natureza geral da actividade e do funcionamento humanos com a actividade e o funcionamento das coisas que construímos e das quais nos rodeámos" (Dick, 2006, p. 31). A ambivalência desta perspectiva reside no facto de, ao reactivar uma certa dimensão mágica, a técnica mais

13 Em sentido próximo ao de Dick, o artista Mark Leckey tem-se apoiado nesta possível noção de tecno-animismo para explorar as suas implicações e estéticas: "The more computed our environment becomes, the further back it returns us to our primitive past, boomerangs us right back to an animistic world view where everything has a spirit, rocks and lions and men. So all the objects in the world become more responsive, things that were once regarded as dumb become addressable, and that universal addressability-a network of things-creates this enchanted landscape. Magic is literally in the air. And that is an altered state, and an endlessly productive one" Leckey \& Cornell (2013).

14 Baudrillard desenvolve assim esta ideia: "En fait, une véritable révolution s'est produite au niveau quotidien: les objets sont devenus aujourd'hui plus complexes que les comportements de l'homme relatifs à ces objets. Les objets sont de plus en plus différenciés, nos gestes le sont en moins en moins. (...) Parce l'objet automatisé marche tout seul, il impose une ressemblance avec l'individu humain autonome, et cette fascination l'emporte. Nous sommes devant un nouvel anthropomorphisme où ce ne sont plus ses gestes, son énergie, ses besoins que I'homme projette sur les objets automatisés, mais l'autonomie de sa conscience, son pouvoir de contrôle, son individualité propre, l'idée de sa personne" (1968, p. 79 e p. 157). 
sofisticada e mais avançada - dentro da qual as tecnologias computacionais ou inteligentes são o seu maior símbolo ${ }^{15}$ poder reencontrar-se com as mais originais formas de relação do humano com o mundo.

Para além deste aspecto, importa ainda o modo como, na passagem referida, K. Dick não destaca de modo particular este ou aquele objecto técnico, mas sobretudo, coloca a enfase no ensemble de todos os objectos na medida em que constituem um ambiente ou uma atmosfera totais, uma infra-estrutura auto-organizada como uma espécie de segunda natureza. Apesar da centralidade que o computador, enquanto objecto específico, virá a adquirir na cultura digital, o que está em causa é sobretudo a extensão das suas lógicas de processamento inteligente e autónomo a qualquer tipo de objecto, seja industrial, doméstico, digital, como um algoritmo, ou de qualquer outra natureza. Neste sentido, a informação, enquanto paradigma tecnológico, parece escapar ao conceito de instrumento porque, mais do que um meio para fins, afirma-se como um meta-medium que integra (ou programa), através da linguagem universal do código numérico, todos os outros meios ou objectos informacionais. A própria ideia de digital poderá não ser considerada como um efeito de um meio particular, mas como um meta-suporte que transforma tudo em medium do processamento técnico. Todos os objectos informacionais evidenciam assim uma recursividade entre si através da qual podem ser ligados. A partir destes princípios operacionaliza-se um mundo povoado por dispositivos autónomos de processamento de informação, isto é, por dispositivos não-humanos aos quais, pela sua capacidade de ler e escrever, ${ }^{16}$ é atribuída inteligência e agenciamento próprio, ao mesmo tempo que, através da sua face virtual, são projectados para o vasto e indefinido conjunto de tudo aquilo que está a converter o mundo em informação e a reconstrui-lo, magicamente, como um novo ambiente artificial.

A concretização material da intuição do tecno-animismo de Philip K. Dick efectivar-se-á a partir do final dos anos 80 do século passado, nomeadamente no Centro de Investigação Xerox em Palo Alto na Califórnia onde Mark Weiser desenvolve o conceito e o programa para uma computação ubíqua:

As tecnologias mais profundas são aquelas que desaparecem. Entrelaçam-se na trama da vida quotidiana até que sejam indistinguíveis dela. (...) A virtualidade dos dados legíveis por computador - todas as diferentes formas pelas quais eles podem ser alterados, processados e analisados - é importada para o mundo físico. (...) Já temos computadores em interruptores de luz, termostatos, aparelhos de som e fornos que ajudam a ativar o mundo. Essas e outras máquinas serão interconectadas numa rede ubíqua (Weiser, 1991, p. 94 e p. 98).

De acordo com Weiser, o computador do século XXI é aquele que é extraído da sua caixa e é desagregado em todo o tipo de objectos quotidianos. Trata-se menos da portabilidade do computador do que da sua integração ubíqua e tendencialmente discreta em ambientes informatizados. Nessa concretização, os computadores também serão menos objectos

15 'The computer is the most animated and intelligent of machines, the most interactive, and by far the least 'mechanical.' Even if we insist upon their entirely mechanical nature, these cybernetic contraptions are now so resilient and complex that they provide us with technological reflections of thought itself, and even life. This potential explains why the electronic computers of the 1950s so quickly gave rise to the notion of artificial intelligence, and why some of today's computer scientists seriously discuss the possibility of breeding life forms made of digital code. Though wisecracking Als have yet to see the light of day, the Internet has already become home to a variety of autonomous and rather parasitic programs - including viruses, Trojan horses, spiders, worms, smartshoppers, and bots - that trawl the Net, replicate themselves, perform various data-processing deeds (often on the sly), and return to their masters with information in tow - that is, if they have masters at all" (Davis, 1998, p. 224).

16 À luz do que atrás referimos a partir de Platão, nesta fase o mundo dos hypomnemata emancipar-se-ia definitivamente do antigo primado da anamnese. Cf. (Bradley, 2011) 
de relações interfaciais destacadas, isoláveis e conscientes, do que uma infra-estrutura que activa as condições do meio envolvente. Na informatização geral dos objectos, o virtual deixa de ser um mundo dentro do computador - fazendo deste um ponto de acesso excepcional -, ou uma simulação circunscrita e dependente de próteses multimédia, para passar a penetrar todos os objectos quotidianos. ${ }^{17}$ Os objectos desaparecem enquanto entes isolados e inertes e fundem-se organicamente com o ambiente; são capazes de comunicar entre si e são sensíveis às condições técnicas e naturais dos meios que os rodeiam e com os quais se sincronizam, sem necessitarem da acção directa dos utilizadores. Nesta transição joga-se um aspecto decisivo: em certa medida, trata-se do fim da descontinuidade entre o mundo físico dos objectos do real e da sua materialidade anónima - e aquele, digamos virtual, do cálculo, da informação e da processualidade técnica. No seu último nível de concretização plena, estaríamos perante uma população em massa de microprocessadores e sensores inteligentes que, como uma nova ecologia informacional, revestiriam a Terra com uma pele electrónica. ${ }^{18}$ Neste derradeiro cenário, seria o próprio planeta, por via da técnica, a ser personificado como um sistema vivo e inteligente.

É neste programa que se funda um conjunto de categorias descritivas que marcam a actualidade, desde logo a categoria de internet das coisas (Easterling, 2012), mas também de cidades sencientes (Shepard, 2011), arquitecturas algorítmicas (Parisi, 2015) ou computação planetária (Bratton, 2015).

\section{A estranheza animista do real}

A conceptualização da autonomia vitalista dos objectos poderá, no entanto, ser apenas parcialmente válida e, perspectivada de um ponto de vista empírico, ser objecto de questionamento: apesar da autonomização e dos algoritmos que governam as trocas informacionais entre objectos, grande parte dos fluxos de informação que são processados e circulam nessa computação ubíqua são produto de comunicação humana, com a sua semântica e significação específicas. Para além disto, esses fluxos de comunicação são produzidos de acordo com uma infra-estrutura e um conjunto de protocolos desenhados estrategicamente também por humanos e, em particular, por companhias tecnológicas. Este último aspecto aponta para o que tem vindo a ser chamado capitalismo cognitivo, o qual pode ser genericamente definido como processo de transformação, ou de reificação, de "qualidades supostamente únicas do trabalho humano - entendido como afectivo, inteligente, astuto e comunicativo - em capital fixo das máquinas" (Srnicek, 2015, p. 308). Se, por um lado, o desenho das estratégias de produção informacional é definido pelos humanos, por outro, a operacionalização dessas estratégias simbólicas é apreendida pelos processos formais e algorítmicos das tecnologias digitais com as suas lógicas que funcionam, em velocidades subliminares e em pura abstracção codificada, para lá das capacidades conceptuais e da fenomenologia humanas. Nesta espécie de aliança intrépida, "a infra-estrutura computacional do capitalismo actual aproxima-se tangencialmente dos limites da física, com as guerras de velocidade do comércio de alta frequência a forçar decisões na escala dos nanossegundos" (Srnicek, 2015, p. 308).

17 "Virtuality is the cultural perception that material objects are interpenetrated by information patterns." (Hayles, 1999, p. 13)

18 Adaptamos aqui o título do artigo de Neil Gross, publicado em 1999 na Business Week, "The Earth Will Don An Electronic Skin": "In the next century, the planet earth will don an electronic skin. It will use the Internet as a scaffold to support and transmit its sensations. This skin is already being stitched together. It consists of millions of embedded electronic measuring devices: thermostats, pressure gauges, pollution detectors, cameras, microphones, glucose sensors, EKGs, electroencephalographs. These will probe and monitor cities and endangered species, the atmosphere, our ships, highways and fleets of trucks, our conversations, our bodies - even our dreams. (...) Think of this as a whole ecology, an information environment that is massively connected" (Gross, 1999). 
A isto se acrescenta uma espécie de curadoria algorítmica, composta por técnicas inteligentes de filtragem, de recomendação personalizada, de desencriptação e de reconhecimento de padrões, nas quais se "combinam a colecção de grandes quantidades de dados sobre os indivíduos (hábitos de compra, padrões de viagens, histórico de navegação, entre outras) com sofisticadas análises matemáticas que fornecem previsões para os profissionais de marketing" (Srnicek, 2015, p. 308). Seja na capitalização da atenção, seja na tipificação e na antecipação de comportamentos por via do rastreamento da produção humana de informação, o emparelhamento entre as operações técnicas e as estratégias de consumo é tão mais eficaz quanto mais antecipável e tipificada for a matriz de acções possíveis dos utilizadores. Perante este horizonte em que a ênfase se coloca nos comportamentos e nas formas simbólicas humanas, a posição tecno-animista parece ser relativizada. Apesar da crescente margem de autonomização da tecnologia e da abstracção das suas processualidades, a sua mobilização prática será em grande medida determinada por estratégias instrumentais que, sob o princípio de um meio para um fim e de acordo com uma certa previsibilidade linear, respondem às necessidades de estratégias e de semióticas fundamentalmente humanas.

Neste sentido, poder-se-á questionar qual é a validade e a pertinência de uma perspectiva como a do tecno-animismo para uma analítica da cultura contemporânea. Erik Davis, num livro dedicado à relação entre a cultura tecnológica e a aquilo que nela persiste de místico e de mágico, formula assim a questão:

Talvez o fenómeno do animismo tecnológico não seja nada mais do que uma actualização recente da sociedade do espetáculo, como um feitiço infantilizante destinado a esmagar qualquer distância crítica que ainda permita a alguns de nós questionar a dominação tecnocapitalista do mundo. Por outro lado, um certo grau de animismo pode também ser visto como uma resposta imaginativamente pragmática e psicologicamente apropriada às qualidades peculiares da selva da informação. (...) O "retorno da magia" pode ser apenas mais uma história de entreter na noite pós-industrial, mas é precisamente por meio dessas histórias que as tecnologias ganham o seu caráter, se não mesmo as suas vidas. (...) A magia também é um mito, mas os mitos dão significado às nossas máquinas (1998, p. 225).

Na posição de Davis, o tecno-animismo e o regresso ou a persistência do mágico são encarados como aquilo a que podemos chamar uma metaforologia prática ou uma metodologia especulativa. No seio desta perspectiva reside uma possibilidade de ultrapassar "a oposição entre cultura e tecnologia, entre homem e máquina", a qual "é falsa e sem fundamento" e apenas "esconde, detrás de um humanismo redutor, uma realidade rica em esforços humanos e em forças naturais, e que constitui o mundo dos objetos técnicos, mediadores entre a natureza e o homem." (Simondon, 2012, p. 9). Em Simondon, a magia, ou mais precisamente aquilo que o filósofo francês chama a fase mágica (2012, pp. 221 - 225), corresponde uma atitude que precede essa oposição, uma disposição epistemológica segundo a qual o homem sustém uma ligação vital com o mundo, numa articulação plena - ou numa indistinção - entre o subjectivo e o objectivo, as figuras e o fundo, o prático e o mitológico, o geográfico e o onírico. A magia corresponde a perspectivar o mundo enquanto realidade reticular constituída por pontos de excepção, isto é, pontos de intensidade excepcional nos quais o poder de acção humana e a capacidade do mundo para influenciar os humanos estão concentrados em potência. Nesta realidade originária, foram os planaltos, os rios, as pedras ou as árvores de grande porte que começaram por concentrar em si esta pulsão na qual a operacionalidade técnica e a projecção estética se sintetizam mutuamente. Mas, mais do que circunscrever a fase mágica a uma historicidade primitiva, irremediavelmente perdida num passado pré-histórico e estuprado pelo progresso da técnica, 
Simondon reconhece que a dimensão mágica da técnica se actualiza sempre que todas estas dimensões - geográfica, natural, técnica, humana - se conjugam de forma reticular na mesma concretização. Essa dimensão mágica é, na verdade, condição e sintoma dos grandes avanços tecnológicos e do nível da sua sofisticação. Duas ilustrações descritas por Simondon são paradigmáticas deste argumento. A primeira relativa à instalação das redes de telecomunicações no final do século XIX:

Não se trata aqui da violação da natureza ou da vitória do ser humano sobre os elementos, porque na verdade são as próprias estruturas naturais que servem de ponto de fixação para a rede que se desenvolve: os pontos de retransmissão dos 'cabos' hertzianos, por exemplo, reúnem-se com os locais elevados da antiga sacralidade, acima dos vales e dos mares. Aqui, a mentalidade técnica completa-se com sucesso e reúne a natureza, tornando-se rede de pensamentos, síntese material e conceptual de particularidade e concentração, individualidade e colectividade - porque toda a força da rede está disponível em cada um dos seus pontos, e os seus labirintos entrelaçam-se com os do mundo, no concreto e no particular. (Simondon, 2009b, p. 22)

E a segunda relativa ao modo como as antenas são indício de uma cosmo-geografia poética na qual o humano se funde com o técnico:

Repare-se numa antena de televisão tal como ela é. (...) É rígida, mas orientada; reparamos que se projecta para longe e que pode receber [sinais] de um emissor distante. Para mim, parece ser mais do que um símbolo; parece representar uma espécie de gesto, um poder quase mágico de intencionalidade, uma forma contemporânea de magia. Neste encontro entre o ponto mais alto e o ponto nodal, que é o ponto de transmissão das hiper-frequências, existe uma espécie de 'co-naturalidade' entre a rede humana e a geografia natural da região. Tem uma dimensão poética, mas também uma dimensão que diz respeito à significação e ao encontro entre significações (Simondon, 2009a, p. 111).

A co-naturalidade a que Simondon se refere pode também ser pensada como uma co-produção entre o humano e a natureza articulada por uma potência estética, isto é, qualquer coisa que é relativa a um excesso ou a um campo de virtualidades que não se esgota em nenhuma concretização técnica particular e que atravessa, com as suas singularidades e os seus traços próprios de expressão, a evolução tecnológica. Podemos então pensar num conceito como o de tecnicidade para nos referirmos a esta tendência, ou seja, a algo que, numa síntese entre o natural, o humano e o técnico, é relativo ao conjunto infinito dos esquemas operacionais que transversalmente actuam na resolução de problemas - o girar, o pendular, o inscrever, o transmitir - a partir e com o próprio mundo.

Dar visibilidade ao modo de existência própria dos objectos técnicos, sejam artefactos, ferramentas, instrumentos, máquinas ou algoritmos, é, abandonando um prisma estritamente antropocêntrico, não apenas reconhecer a forma como a génese da cultura humana é sempre uma tecnogénese, como também promover uma atenção sobre as materialidades e as processualidades que determinam a experiência, na maior parte dos casos de forma inconsciente. Sobre este aspecto podemos recorrer à metáfora do espelho que o teórico do design Brendan Hookway (2014) apresenta: na mesma medida em que encontramos sempre o reflexo da nossa imagem, e é nela que nos concentramos, antes de encontrarmos o próprio espelho enquanto objecto material, também nos detemos nos usos simbólicos e semióticos que 
extraímos da tecnologia, naturalizando-a sobre um plano de transparência e de imeaditicidade que oculta os agenciamentos maquínicos através dos quais as nossas interacções são desenhadas. Como resposta, será produtivo o exercício que artificializa - e que reencanta - as tecnologias que constituem o nosso quotidiano.

Entre a legitimação do estatuto cultural da técnica e a consciencialização do seu papel enquanto a priori da experiência encontra-se também a possibilidade de desvio dos protocolos e da previsibilidade que ditam que apenas um conjunto de funcionalidades ou usos específicos, muitas vezes desenhados sob estratégias de rentabilização, sejam possíveis. A magia ou o reencantar ligam-se à contingência, à indeterminação e ao ruído, abrindo uma via especulativa para contemplar como "os graus de incerteza podem ser produtivos: ao interromper a previsibilidade linear, a incerteza pode ampliar o espectro cognitivo dos actores (humanos e não humanos) envolvidos nas interacções" (Marenko \& Allen, 2016, p. 2). Haverá sempre outras hipóteses de operacionalização e de concretização da técnica para além desses protocolos.

Em suma, o tecno-animismo pode ser encarado como uma metodologia especulativa ${ }^{19}$ que, por projectar nos objectos técnicos uma vida própria, Ihes inscreve uma alteridade e uma estranheza que potencia novas perspectivas para além dos usos e das relações habituais que se dão por garantidas e que escondem os objectos, assim como as estratégias políticas e comerciais que os mobilizam e rentabilizam. Reencantar os objectos técnicos é, por um lado, criar uma possibilidade para os suspender dos seus fluxos instrumentais e previsíveis e, por outro, instalar um realismo mágico que perturba as articulações de uma realidade mera e exclusivamente humana (Morton, 2013). Mais do que uma coincidência entre as perspectivas subjectivamente construídas e uma suposta realidade dos fenómenos em si mesmos, esta revisão da noção de realismo abre a possibilidade para um real que terá sempre uma vibração própria para além da capacidade humana de o apreender. À luz deste princípio, também o animismo não representará a mera atribuição de traços antropomórficos a realidades não-humanas, mas, inversamente, corresponderá à suspeita encantada de que tanto no mundo técnico, como no vegetal e no mineral estão ao trabaIho - com ritmos e escalas díspares - agências que activam, determinam ou limitam a infra-estrutura da acção humana. Esta viragem não implica, em todo o caso, uma anulação da dimensão humana na experiência das interacções possíveis. Trata-se de a especificar e enquadrar nas complexas e dinâmicas redes híbridas de máquinas, formas biológicas e cognitivas e processos culturais que, em conjunto, mas também de forma particular, estão a produzir. A virtude de uma tal metodologia especulativa, para adoptarmos uma passagem de Quentin Meillassoux, "não está em tornar o realismo ilusório, mas sim em torná-lo surpreendente, aparentemente impensável, mas verdadeiro e, como tal, eminentemente problemático" (2006, p. 38).

Mais do que a suspeita de um retrocesso civilizacional, no reencontro da pulsão animista e da estranheza problemática do real está uma outra suspeita: aquela que atravessa o olhar próprio das crianças e que projecta um mundo que, ao mesmo tempo que é fonte de espanto e surpresa constantes,

19 "Animistic design as a strategic and experimental tool to rethink human-machine interaction. Deployed as a speculative method of investigation, animism is a post-cognitive framework that produces new fictions and fosters new myth-making narratives. Animism offers a way of thinking about interaction differently: neither from the perspective of the user, nor from the perspective of the object but from the ongoing modulation of their less-than-predictable interaction" (Marenko \& Allen, 2016. p. 2). 
pode sempre ser recriado por via do brincar, exercício no qual o prático e o especulativo se conjugam na perfeição. ${ }^{20}$

\section{References}

Anders, G. (1961, [1957]). Commandments in the Atomic Age. In G. Anders, Burning Conscience. Monthly Review Press.

Arendt, H. (1998, [1958]). The Human Condition. The University of Chicago Press.

Aristotle. Politics. (The Loeb Classical Library, No. 264). Harvard University Press

Aristotle. The Physics, Vol. I, Books I-IV. (The Loeb Classical Library, No. 228). Harvard University Press

Baudrillard, J. (1968). Le Système des Objets. Gallimard.

Bennett, J. (2015). Systems and Things - On Vital Materialism and Object-Oriented Philosophy. In Grusin, R. (Ed.) The Nonhuman Turn. University of Minnesota Press.

Bierce, A. (2011, [1899]). Moxon's Master. In Ambrose Bierce: The Devil's Dictionary, Tales \& Memoirs, 252 - 261. The Library of America.

Bradley, A. (2011) Originary Technicity: The Theory of Technology from Marx to Derrida. Palgrave Macmillan.

Bratton, B. (2015). The Stack - On Software and Sovereignty. The MIT Press.
Bryant, L. (2014) Onto-Cartography - An Ontology of Machines and Media. Edinburgh University Press.

Connor, S. (2016). How to do Things with Writing Machines. In Sean Pryor and David Trotter (Eds.) Writing, Medium, Machine: Modern Technographies, 18 - 34. Open Humanities Press.

Davis, E. (1998) TechGnosis - Myth, Magic and Mysticism in the Age of Information. Serpents Tail.

Deleuze, G. \& Guattari, F. (1980) Mille Plateaux - Capitalisme et Schizophrénie 2. Les Éditions de Minuit.

Dick, P. K (2006 [1972]). O androide e o humano. Vega.

Diderot, D. (1753). Pensées sur l'interprétation de la nature. (Epub) Livre France.

Easterling, K. (2012). An Internet of Things. In A. Vidokle Et al. (Eds.) The Internet Does Not Exist - E-flux Journal. Sternberg Press.

Essinger, J. (2004). Jacquard's Web: How a Hand-Loom Led to the Birth of the Information Age. Oxford University Press.

Flusser, V. (2010) Uma Filosofia do Design - a Forma das Coisas. Relógio d'Água.

Gell, A. (1988) Technology and Magic. Anthropology Today, Vol. 4, No. 2 (Apr.), 6 - 9

20 "Magic consists of a symbolic commentary on technical strategies in production, reproduction, and psychological manipulation. I suggest that magic derives from play. When children play, they provide a continuous stream of commentary on their own behaviour. This commentary frames their actions, divides it up into segments, defines momentary goals, and so on. It seems that this superimposed organizational format both guides imaginative play as it proceeds, and also provides a means of internalizing it and recalling it, as well as raw materials for subsequent exercises in innovation and recombination, using previously accumulated materials in new configurations. (...) The same is true of magic, which sets an ideal standard, not to be approached in reality, towards which practical technical action can nonetheless be oriented" (Gell, 1988, p. 8). 
Gell, A. (1992) The Technology of Enchantment and the Enchantment of Technology. In J. Coote and A. Shelton (Eds.) Anthropology, Art and Aesthetics. pp. 40-66. Clarendon.

Gross, N. (1999) The Earth Will Don an Electronic Skin. Business Week. August 30. http://www.businessweek. com/1999/99_35/b3644024.htm.

Hayles, K. N. (1999) How We Became Posthuman - Virtual Bodies in Cybernetics, Literature, and Informatics. University of Chicago Press.

Hilpinen, R. (2004). "Artifact," The Stanford Encyclopedia of Philosophy (Fall 2004 Edition). http://plato.stanford.edu/archives/fall2004/entries/artifact

Hookway, B. (2014). Interface. The MIT Press.

Latour, B. (1988). Irreductions. In B. Latour The Pasteurization of France. Harvard University Press.

Lazzarato, M. \& Melitopoulos, A. (2010). Machinic Animism. In A. Franke (Ed.) Animism. Sternberg Press.

Leckey, M. \& Cornell, L. "Techno-animism". Mousse Magazine 37. http://moussemagazine.it/lauren-cornell-techno-animism-2013/

Marx, K. (1990, [1867]). Capital: A Critique of Political Economy, Vol. I. Penguin Books.

Marenko, B. \& Allen, P. V. (2016). Animistic design: how to reimagine digital interaction between the human and the nonhuman. Digital Creativity. DOI: 10.1080/14626268.2016.1145127

Meillassoux, Q. (2006). Après la finitude - Essai sur la necessite de la contingence. Seuil.
Morton, T. (2013). Realist Magic Objects, Ontology, Causality. Open Humanities Press.

Novalis, F. (1997, [1799]). Christendom or Europe? In Philosophical Writings, 137-52. State University of New York Press.

Parisi, L. (2015). Automated Architecture: Speculative Reason in the Age of the Algorithm. In C. Cox, J. Jaskey and S. Malik (Eds.) Realism Materialism Art. Stenberg Press.

Poe, E. A. (2015, [1836] O Jogador de Xadrez de Maelzel. In Todos os Contos. Círculo de Leitores.

Plato. Phaedrus. (Loeb Classical Library). Harward University Press.

Rodrigo, P. (2014) Sur l'Ontologie de Marx - Auto-production, Travail Aliéné et Capital. Paris: Vrin.

Rose, D. (2015). Enchanted Objects: Innovation, Design, and the Future of Technology. Scribner.

Serres, M. (1995, [1982). Genesis. The University of Michigan Press.

Simondon, G. (2009a, [1968]). Entretien sur la méchanologie. Revue de synthèse 130(1), 103 - 32.

Simondon, G. (2012, [1958]). Le Mode D'Existence des Objets Techniques. Aubier.

Simondon, G. (2009b). Technical Mentality. Parrhesia 7, 17 - 27.

Singleton, B. (2015). "(Notes Towards) Speculative Design". https://shifter-magazine.com/wp-content/uploads/2015/09/ Singleton-Notes-Towards-Speculative-Design.pdf 
INTERNATIONAL JOURNAL OF FILM AND MEDIA ARTS (2021) Vol. 6, №. 1

Shepard, M. (2011). Sentient City - Ubiquitous Computing, Architecture, and the Future of Urban Space. The MIT Press.

Srnicek, N. (2015). Computational Infrastructures and Aesthetics. In C. Cox, J. Jaskey and S. Malik (Eds.) Realism Materialism Art. Stenberg Press.

Stiegler, B. (1998, [1994]). Technics and Time 1 - The Fault of Epimetheus. Stanford University Press.

Weiser, M. (1991). The computer for the $21^{\text {st }}$ Century. Scientific American 265(3), 94 - 104.

Wiener, N. (1966) God and Golem, Inc. - A Comment on Certain Points where Cybernetics Impinges on Religion. The Mit Press.

Wolfe, J. (2004). Humanism, Machinery, and Renaissance Literature. Cambridge University Press. 